\title{
Use of Silk Protein, Sericin, as a Sustained-Release Material in the Form of a Gel, Sponge and Film
}

\author{
Ayumu Nishida, ${ }^{*, a}$ Masaki Yamada,${ }^{a}$ Takanori Kanazawa, ${ }^{b}$ Yuuki TaKashima, ${ }^{b}$ Kiyohisa Ouchi, ${ }^{a}$ and \\ Hiroaki OKADA ${ }^{b}$ \\ ${ }^{a}$ Pharmaceutical Technology Laboratories, Kissei Pharmaceutical Co., Ltd.; 4365-1 Kashiwabara, Hotaka, Azumino \\ 399-8304, Japan: and ${ }^{b}$ Department of Pharmaceutics and Drug Delivery, School of Pharmacy, Tokyo University of \\ Pharmacy and Life Sciences; 1432-1 Horinouchi, Hachioji, Tokyo 192-0392, Japan.
}

Received May 30, 2010; accepted August 17, 2010; published online August 25, 2010

To evaluate the usability of silk protein (sericin, SC) as a sustained-release material, the physicochemical properties of SC and the release profiles of model drugs from SC gel, sponge and film were studied. Heat aids the dissolution of SC. The molecular weight of SC tended to decrease as the heating temperature and heating time increased. The gel and sponge formed by SC were moldable and consisted of high molecular weight SC polymers $(250 \mathrm{kDa}$ and about $400 \mathrm{kDa})$. SC film was easily broken and exhibited elastic distortion. The addition of moisture-retaining plasticizer (glycerin and sorbitol) improved the film-forming characteristics of SC. The results suggested that $\mathrm{SC}$ is practical as a moldable gel and sponge, and as a tensible film. To evaluate the release profiles of small molecules, fluorescein isothiocyanate-dextran ((1) FD4, $4 \mathrm{kDa}$ and (2) FD70, $70 \mathrm{kDa}$ ) were used as two model drugs with significantly different molecular weights, and fluorescein isothiocyanate-albumin ((3) FA, $66 \mathrm{kDa}$ ) was used as a charged drug. Each was formulated in SC gel, sponge and film. In each preparation, the release rate of the model drugs tended to be $\mathrm{FA}<$ FD70 $<$ FD4. FA, which has a large molecular weight and negative charge, was released for the longest period of time ( $\geq 1$ week) from each preparation. The results suggest that SC is usable as an aqueous sustained-release material for high molecular weight drugs. Furthermore, if the drug is charged, its release can be sustained for an extended time.

Key words sericin; sustained release; film; gel; sponge

Sericin (SC) is a component of silk protein in the silkworm cocoon. Silk protein consists of fibroin as the fiber and $\mathrm{SC}$ as the glue. SC comprises $25-30 \%$ of the silkworm (Bombyx mori) cocoon. ${ }^{1)}$ Although $\mathrm{SC}$ is composed of 18 different amino acids, it contains a high number of polar side chains with hydroxyl, carboxyl and amino groups. ${ }^{1)}$ Isolation and characterization of SC components from the cocoon of Bombyx mori showed that SC primarily consists of three polypeptides with molecular weights of 150, 250 and 400 $\mathrm{kDa}$. The phenylthiocarbamyl (PTC) method $^{2}$ showed that the amino acid compositions of all three SC have high contents of serine (Ser, 33.2-39.0\%), glycine (Gly, 14.1$16.0 \%$ ) and aspartic acid/asparagine (Asp/Asn, 11.3$15.7 \%$ ). The structural analysis and cloning of SC genes Ser 1 and Ser2 (Src-2) have been described. ${ }^{3-6)}$ Correspondence of the amino acid composition of $\mathrm{SC}$ with these genes suggested that the 150 - and $400-\mathrm{kDa}$ SCs correspond to Ser1 proteins $(77-331 \mathrm{kDa})$ encoded by the Ser 1 gene, and that the $250-\mathrm{kDa} \mathrm{SC}$ corresponds to $\mathrm{S}-2$ protein $(227 \mathrm{kDa})$ encoded by the $S r c-2$ gene. $\left.{ }^{2}\right)$ The most abundant component is the largest $\mathrm{SC}(400 \mathrm{kDa})$, which corresponds to the Ser1C protein $(331 \mathrm{kDa}){ }^{2}{ }^{2}$ A repetitive 38 -amino acid sequence rich in Ser $(40 \%)$ dominates a large part of the Ser1C protein and is predicted to have a strong tendency to form $\beta$-sheet structure. Another part of the Ser1C protein is hydrophilic and has a high content of charged residues including acidic (glutamic acid (Glu) and Asp) and basic (lysine (Lys) and arginine (Arg)) amino acids. ${ }^{6}$ In contrast, the $250-\mathrm{kDa}$ SC polypeptide, which corresponds to S-2 protein $(227 \mathrm{kDa})$, has less $\beta$ sheet forming propensity and higher hydrophilicity than the $150-$ and $400-\mathrm{kDa}$ polypeptides. This is because the $250-\mathrm{kDa}$ $\mathrm{SC}$ contains larger amounts of $\beta$-sheet breaking residues like Glu, glutamine (Gln) and Lys, and smaller amounts of $\beta$ - sheet favoring residues like threonine (Thr) and tyrosine (Tyr) compared to the 150- and 400-kDa SCs. ${ }^{2}$

Historically, sericulture was widely carried out to support the silk spinning and weaving industry in Japan. SC was degraded into low molecular weight fragments by a silk scouring process and released as a waste product. ${ }^{1)}$ However, recent progress in transgenic technology has produced strains of transgenic silkworms that secrete recombinant proteins such as vaccines and cytokines in the SC of their cocoons. ${ }^{7,8)}$ Furthermore, the modification of silk protein properties by transgenic silkworms is now being attempted. Takabayashi reported the applicability of silk fibroin, expressed with a cell attachment factor, in the construction of biocompatible and biodegradable artificial blood vessels. ${ }^{9,10)}$ Thus, modified silk protein as a functional material and a generator of recombinant protein is a promising new direction in the field of sericulture.

In a study of the application of SC as a biomaterial, SC was reported to enhance the attachment of cultured human skin fibroblasts. ${ }^{11)}$ In addition, SC hydrogel sheets containing a mixture of water and ethanol, as well as SC cream, hold promise as practical dressings for wound healing. ${ }^{12,13)} \mathrm{SC}$ has moisturizing, antioxidant and UV-resistant effects ${ }^{1,14-16)}$ and is expected to have highly biocompatible properties.

For injection and implantation, several other biocompatible and biodegradable sustained-release materials are being or have already been developed. For example, synthetic polymers such as poly (lactic-co-glycolic acid) (PLGA) and natural polymers like gelatin and chitosan are being investigated. ${ }^{17-20)}$ Although these biocompatible and biodegradable materials have excellent characteristics and are indispensable as drug delivery systems, they suffer from several potential drawbacks including poor compatibility with certain drugs, 
the inevitable need to use large amounts of organic solvent and toxic cross-linker during their preparation, and dangers related to bovine spongiform encephalopathy (BSE). It is thus necessary to widen the number of biocompatible sustained-release materials in the near future. Although SC in various forms may be a promising biocompatible material, drug release from SC materials, the effect of the physical state of the SC material, the molecular weight of SC, and the effect of the characteristics of the drugs all remain largely unstudied.

The present study aimed to use SC as an aqueous sustained-release material for the preparations of gel, sponge and film practical for use as medical dressing films, ointment bases, injectable gels, and implants. First, the physicochemical properties of SC, such as molecular weight and solubility, the moldablity of SC gel and sponge, and the tensibility of SC film were evaluated. SC film was found to break easily and exhibited elastic distortion, making its use impractical. Improvement of the film-forming properties and tensibility of SC film by the addition of plasticizers such as glycerin (GL), sorbitol (ST) and polyethylene glycol (PG) were examined. Furthermore, to evaluate the usability of SC as a sustained-release material, the release properties of three model drugs with different molecular weights and charge were measured in SC gel, sponge and film.

\section{Experimental}

Materials Sericin Hope SHC (SC purity is 98.5\%, Kougensha Co., Ltd., Nagano, Japan) was used as the source of intact SC. Fluorescein isothiocyanate-dextran (FD4, 4 kDa and FD70, $70 \mathrm{kDa}$ ) were used as model drugs having different molecular weights, and fluorescein isothiocyanate-albumin (FA, 66 kDa) was used as a charged model drug (Sigma-Aldrich Japan Co., Ltd., Tokyo, Japan). Glycerin (GL, Wako Pure Chemicals Industries Ltd., Osaka, Japan), D-sorbitol (ST, Mitsubishi-Kagaku Foods Co., Ltd., Tokyo, Japan) and PEG-400 (PG, Wako Pure Chemicals Industries Ltd.) were used as plasticizers in the SC films.

Preparation of SC Solution, Gel, Sponge and Film Table 1 shows the conditions used to prepare the SC solutions (SCS-1, SCS-2, SCS-3 and SCS-4). SC ( 1 or $2 \%$ ) was dissolved in purified water using various temperatures and heating times $\left(100^{\circ} \mathrm{C}\right.$ for $20 \mathrm{~min}, 100^{\circ} \mathrm{C}$ for $60 \mathrm{~min}$ and $120^{\circ} \mathrm{C}$ for $20 \mathrm{~min}$ ). An autoclave apparatus (HA300M5, Hirayama Manufacturing Corporation, Saitama, Japan) was used to dissolve SC at $120^{\circ} \mathrm{C}$. As SC solutions contain some insoluble SC and other particulates, each SC solution was centrifuged at 3000 rotations/min for $5 \mathrm{~min}$ to remove the insoluble material, then cooled to room temperature.

To evaluate the physicochemical properties of the SC preparations, SC gel, sponge and film were made from these SC solutions. To prepare SC gel, each $\mathrm{SC}$ solution was kept at $5^{\circ} \mathrm{C}$ for $1 \mathrm{~d}$. To prepare insolubilized SC sponge, each SC solution was frozen at $-80^{\circ} \mathrm{C}$ for $1 \mathrm{~d}$ to cause ice nuclei to form and grow, resulting in dehydration of the SC phase. ${ }^{21)} \mathrm{SC}$ sponge was thawed at room temperature for about $30 \mathrm{~min}$ just before use. To prepare SC film, each SC solution was cast on a polyethylene dish and dried. To improve the film-forming properties and tensibility of SC film, the effect of the addition of plasticizer (GL, ST and PG) was examined. Table 2 shows the formulations of the SC films containing each plasticizer. ST, GL or PG was dis-

Table 1. Preparation Conditions of the SC Solutions

\begin{tabular}{ccccc}
\hline \hline $\begin{array}{c}\text { Sample } \\
\text { solution }\end{array}$ & $\begin{array}{c}\mathrm{SC}^{a)} \\
(\%)\end{array}$ & $\begin{array}{c}\text { Heating } \\
\text { temperature } \\
\left({ }^{\circ} \mathrm{C}\right)\end{array}$ & $\begin{array}{c}\text { Heating } \\
\text { time } \\
(\mathrm{min})\end{array}$ & Medium \\
\hline SCS-1 & 1 & 100 & 20 & Purified \\
SCS-2 & 1 & 100 & 60 & water \\
SCS-3 & 1 & 120 & 20 & \\
SCS-4 & 2 & 100 & 20 & \\
\hline
\end{tabular}

a) Concentration of SC powder loaded into water. solved into SCS-4 to a final concentration of $0.25,0.5$ and $1.0 \%$. SCS-4 $(40 \mathrm{ml})$ with ST, GL or PG, or without any plasticizer, were each cast on $10 \mathrm{~cm} \times 10 \mathrm{~cm}$ polyethylene dishes and dried at $25^{\circ} \mathrm{C} / 30 \% \mathrm{RH}$ for $1 \mathrm{~d}$. The dried films were used after aging at $25^{\circ} \mathrm{C} / 60 \% \mathrm{RH}$.

To evaluate their drug release properties, SC gel, sponge and film individually containing one of the three model drugs (FD4, FD70 or FA) were prepared. Table 3 shows the formulations of SC gels, sponges and films containing each model drug. Each model drug was dissolved in SCS-1 $(0.5 \mathrm{mg} / \mathrm{ml})$. SCS-1 containing each model drug $(2 \mathrm{ml})$ was dispensed into a cylindrical glass vessel with an inner diameter of $23 \mathrm{~mm}$. To prepare SC films (F-FD4, F-FD70 and F-FA), SCS-1 containing each drug was dried at $40{ }^{\circ} \mathrm{C}$ for $1 \mathrm{~d}$ in the vessels. To prepare SC gels (G-FD4, G-FD70 and G-FA), SCS-1 containing each drug was cooled at $5^{\circ} \mathrm{C}$ for $1 \mathrm{~d}$ in the vessels and brought to room temperature before use. To prepare SC sponge (S-FD4, SFD70 and S-FA), SCS-1 containing each drug was frozen at $-80^{\circ} \mathrm{C}$ for $1 \mathrm{~d}$ in the vessels and the sponge was formed by thawing the preparation at room temperature for about $30 \mathrm{~min}$ and removing the free water just before use.

Physico-Chemical Properties. Determination of Molecular Weight of SC The molecular weight of SC dissolved under various heating conditions (SCS-1, SCS-2 and SCS-3 in Table 1) was determined by sodium dodecyl sulfate-polyacrylamide gel electrophoresis (SDS-PAGE). The SC solutions were electrophoresed on $10 \%$ polyacrylamide gels (C10L, ATTO Corporation, Tokyo, Japan) and the proteins were stained with Coomassie Brilliant Blue R-250.

Solubility of SC The concentration of SC dissolved under various heating conditions (SCS-1, SCS-2 and SCS-3 in Table 1) was obtained by directly measuring the weight of the solid content after drying each SC solution.

Table 2. Formulations of SC Film with and without Plasticizer

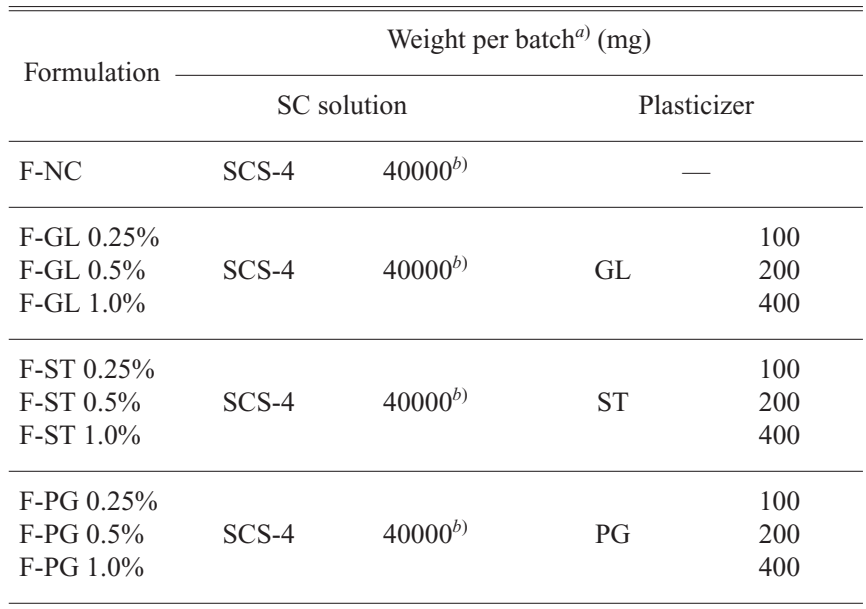

a) SCS-4 films with plasticizer and without were cast on $10 \mathrm{~cm} \times 10 \mathrm{~cm}$ polyethylene dishes. b) Water was removed by a drying process.

Table 3. Formulations of SC Gel, Sponge and Film Containing Model Drugs

\begin{tabular}{|c|c|c|c|c|c|}
\hline \multirow{2}{*}{\multicolumn{2}{|c|}{ Formulation }} & \multicolumn{4}{|c|}{ Weight per unit (mg) } \\
\hline & & \multicolumn{2}{|c|}{ SC solution } & \multicolumn{2}{|c|}{ Model drug } \\
\hline SC gel & $\begin{array}{l}\text { G-FD4 } \\
\text { G-FD70 } \\
\text { G-FA }\end{array}$ & SCS-1 & 2000 & $\begin{array}{l}\text { FD4 } \\
\text { FD70 } \\
\text { FA }\end{array}$ & $\begin{array}{l}1 \\
1 \\
1\end{array}$ \\
\hline SC sponge & $\begin{array}{l}\text { S-FD4 } \\
\text { S-FD70 } \\
\text { S-FA }\end{array}$ & SCS-1 & 2000 & $\begin{array}{l}\text { FD4 } \\
\text { FD70 } \\
\text { FA }\end{array}$ & $\begin{array}{l}1 \\
1 \\
1\end{array}$ \\
\hline SC film & $\begin{array}{l}\text { F-FD4 } \\
\text { F-FD70 } \\
\text { F-FA }\end{array}$ & SCS-1 & $2000^{a)}$ & $\begin{array}{l}\text { FD4 } \\
\text { FD70 } \\
\text { FA }\end{array}$ & $\begin{array}{l}1 \\
1 \\
1\end{array}$ \\
\hline
\end{tabular}

a) Water was removed by a drying process. 
Molding Property of SC Gel and Sponge To evaluate the molding property of SC gel and sponge, the appearance of SC gels and sponges was observed visually and by scanning electron microscopy (SEM, JSM-5200LV, JEOL, Tokyo, Japan). The compressive strength of SC gels was measured with a RHEO METER CR200D (Sun Scientific Co., Ltd., Tokyo, Japan). SC gels were compressed in cylindrical vessels with an inner diameter of $30 \mathrm{~mm}$ and with a depth of $20 \mathrm{~mm}$ at a speed of $40 \mathrm{~mm} / \mathrm{min}$ using a disk $20 \mathrm{~mm}$ in diameter.

Film-Forming Properties and Tensibility of SC Films To evaluate the film-forming properties of SC, the surface structure of SC films was observed by SEM. The elongation and tensile strength of SC films with GL, ST or PG, and without plasticizer, were measured. Based on the test conditions of Teramoto et al., ${ }^{22)} \mathrm{SC}$ films were cut into $20 \mathrm{~mm} \times 5 \mathrm{~mm}$ pieces, set with a gauge length of $10 \mathrm{~mm}$, and stretched at $20 \mathrm{~mm} / \mathrm{min}$ in a RHEO METER CR200D. The elongation and tensile strength of moistened SC films was measured by the same methods used for dried SC films 2 min after spraying with phosphate-buffered saline (PBS, $\mathrm{pH} 7.4){ }^{22)}$

Moisture Content of SC Films The moisture content of SC films was measured by loss on drying (LOD) using an IR Moisture Determination Balance (FD-610, Kett, Tokyo, Japan) following drying at $120^{\circ} \mathrm{C}$ for $20 \mathrm{~min}$.

Attenuated Total Reflection-Fourier Transform Infrared Spectroscopy (ATR-FT-IR) Analysis of SC Films To analyze the secondary structure of SC protein, ATR-FT-IR spectra of the surface of SC films with GL, ST or PG, and without plasticizer, were obtained using an ATR-FT-IR spectrometer (IR Prestige-21, Shimadzu Corporation, Kyoto, Japan) at a resolution of $4 \mathrm{~cm}^{-1}$. All samples and backgrounds were scanned 45 times ATR measurements were performed using a single-reflection diamond ATR attachment (DurasamplIR II, SensIR Technologies, Danbury, CT, U.S.A.).

Release Profiles of Model Drugs from SC Gel, Sponge and Film SC gel, sponge and film (respective thicknesses, $50 \mathrm{~mm}, 50 \mathrm{~mm}$ and 30 $70 \mu \mathrm{m}$ ) containing one of the model drugs (FD4, FD70 or FA) were placed in test tubes (23-mm inner diameter) for the dissolution tests (Table 3). PBS $(5 \mathrm{ml}, \mathrm{pH} 7.4)$ was added in the test tubes and the contents were incubated at $37^{\circ} \mathrm{C}$

To measure the release rate of FD4 and FD70 from SC preparations in PBS, fluorescence absorbance (Ex 493 nm, Em $515 \mathrm{~nm}$ ) of released FD4 and FD70 was detected with time using a fluorescence spectrophotometer (Spectra Max, Molecular Devices, Sunnyvale, CA, U.S.A.). To measure the release rate of FA from SC preparations in PBS, UV absorbance of released FA was detected at $495 \mathrm{~nm}$ with time using a UV spectrophotometer (UV2500PC, Shimadzu Corporation, Kyoto, Japan).

\section{Results and Discussion}

Physico-Chemical Properties. SC Solution Four different SC solutions were obtained by dissolving SC in purified water using various conditions of temperature and heating time (Table 1). Two polypeptide bands, one of $250 \mathrm{kDa}$ and one of higher molecular weight, were mainly observed in SCS- $1\left(100^{\circ} \mathrm{C}\right.$ for $\left.20 \mathrm{~min}, \mathrm{~A}\right)$ and SCS-2 $\left(100^{\circ} \mathrm{C}\right.$ for $60 \mathrm{~min}$, $\mathrm{B})$, and a diffuse band around $100 \mathrm{kDa}$ was observed in SCS$3\left(120^{\circ} \mathrm{C}\right.$ for $\left.20 \mathrm{~min}, \mathrm{C}\right)$ by SDS-PAGE (Fig. 1). The bands at $250 \mathrm{kDa}$ and the larger molecular weight band correspond approximately to the $180-$ to $250-\mathrm{kDa}$ and $400-\mathrm{kDa}$ bands of intact SC reported by Takasu et $a l^{2)}$ and Teramoto et al. ${ }^{23)}$ The bands obtained from SCS-2 (B) and particularly from SCS-3 (C) tended to become diffuse, indicating a wide distribution in molecular weight and the presence of smaller molecules, suggesting that SC degraded into lower molecular weight fragments with increasing temperature and heating time. This is consistent with the results of Teramoto et al., who suggested, based on ${ }^{13} \mathrm{C}$-NMR spectroscopy data, that SC Asp residues might be hydrolyzed preferentially during heat treatment. ${ }^{24)}$ In addition, Mase et al. reported that hydrolytic degradation of SC in solution occurred between $110^{\circ} \mathrm{C}$ and $120^{\circ} \mathrm{C}^{25)}$

The concentration of dissolved SC polypeptides (SCS-1, SCS-2 and SCS-3) was respectively about $0.6,0.8$ and $1 \%$ (Fig. 2). Because SC was degraded by heat in the order SCS-
$1<$ SCS-2 $<$ SCS-3 (Fig. 1), degraded SC apparently dissolves, increasing the concentration of dissolved SC components. The concentration of dissolved SCS-4 was about $1.2 \%$. Therefore, to obtain SC with predictable concentration and molecular weight, the heating conditions used to dissolve $\mathrm{SC}$ should be regulated.

SC Gel and Sponge The moldability of SC gels and sponges made from various SC solutions (SCS-1, SCS-2 and SCS-3) indicated that SC gel and sponge from SCS-1 and SCS-2 tended to maintain their original shapes better than SCS-3 (Figs. 3A, B). Furthermore, the compressive strength

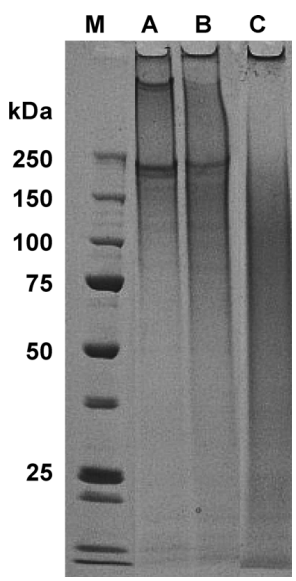

Fig. 1. SDS-PAGE Patterns of SC Dissolved under Various Dissolution Conditions (A: $100^{\circ} \mathrm{C}$ for $20 \mathrm{~min}$; B: $100^{\circ} \mathrm{C}$ for $60 \mathrm{~min}$; C: $120^{\circ} \mathrm{C}$ for $20 \mathrm{~min}$ )

M: Molecular weight markers.

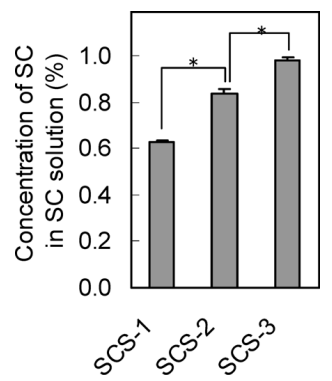

Fig. 2. Concentration of SC Dissolved under Various Dissolution Conditions (SCS-1: $100^{\circ} \mathrm{C}$ for $20 \mathrm{~min}$; SCS-2: $100^{\circ} \mathrm{C}$ for $60 \mathrm{~min}$; SCS-3: $120^{\circ} \mathrm{C}$ for $20 \mathrm{~min}$ )

Solutions were prepared from $1 \%(w / w)$ SC in water. Statistical comparison was made by two-sided $t$-test $(a=0.05) . * p<0.01$.

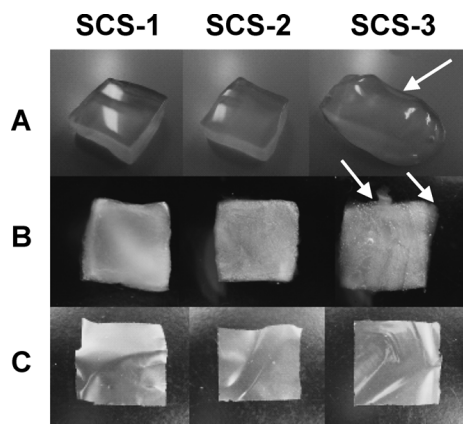

Fig. 3. Photographs of SC Gel (A), Sponge (B) and Film (C) from SC Solutions Prepared Using the Various Dissolution Conditions (SCS-1: $100^{\circ} \mathrm{C}$ for $20 \mathrm{~min}$; SCS-2: $100^{\circ} \mathrm{C}$ for $60 \mathrm{~min}$; SCS-3: $120^{\circ} \mathrm{C}$ for $20 \mathrm{~min}$ )

Arrowhead: deformed part in the gel or sponge. 
of SC gel was SCS-3<SCS-2 $<$ SCS-1 (Fig. 4). Analysis of the amino acid composition of SC showed that high molecular weight SC (400 kDa) corresponds to Ser1C protein coded by the Ser1 gene. ${ }^{2)}$ A repetitive 38 -amino acid sequence rich in Ser dominates a large part of Ser1C and is predicted to have high propensity for forming $\beta$-sheet structures with strong intermolecular hydrogen bonds, thereby producing the network structure of the hydrogel. ${ }^{6}$ In this study, the amount of high molecular weight SC $(400 \mathrm{kDa})$ increased in the order SCS-3 $<$ SCS-2 $<$ SCS-1 (Fig. 1), suggesting that gels containing larger amounts of $400-\mathrm{kDa} \mathrm{SC}$ withstood higher mechanical stress better than gels made from lower molecular weight SC. The results indicate that SCS-1 (dissolution condition: $100^{\circ} \mathrm{C}$ for $20 \mathrm{~min}$ ) is suitable for the preparation of moldable materials with enough mechanical strength to allow study of the release of model drugs.

SC Film SC films were prepared from SCS-1, SCS-2, SCS-3 and SCS-4 (Table 1). Films made of SC alone exhibited elastic distortion and were easily broken during the drying process, showing that their use is not practical (Fig. 3C). To improve the film-forming properties and tensibility of SC film, GL, ST or PG were added as a plasticizer (Table 2). Figure 5 shows SEM photographs of dried SC films with GL (A), ST (B) or PG (C), and without plasticizer (D). Although SC film without plasticizer exhibited elastic distortion and easily cracked, the SC films containing GL, ST or PG exhibited improved film-forming properties (Fig. 5).

ATR-FT-IR analysis was used to analyze the change in SC film properties. The amide absorption of FT-IR is influenced sensitively by the secondary structure of the protein, and amide I absorption (IR $\mathrm{cm}^{-1}: 1700-1600$ region) is the most useful band for the determination of secondary structure. ${ }^{26}$ Amide I absorption primarily represents the $\mathrm{C}=\mathrm{O}$ stretching vibration of the amide group. ${ }^{26)}$ ATR-FT-IR analy-

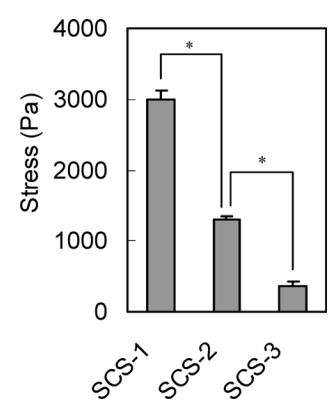

Fig. 4. Compressive Strength of SC Gels from SC Solutions Prepared Using the Various Dissolution Conditions (SCS-1: $100^{\circ} \mathrm{C}$ for $20 \mathrm{~min}$; SCS2: $100^{\circ} \mathrm{C}$ for $60 \mathrm{~min}$; SCS-3: $120^{\circ} \mathrm{C}$ for $20 \mathrm{~min}$ )

Each bar represents the mean \pm S.D. $(n=3)$. Statistical comparison was made by twosided $t$-test $(a=0.05) . * p<0.01$.

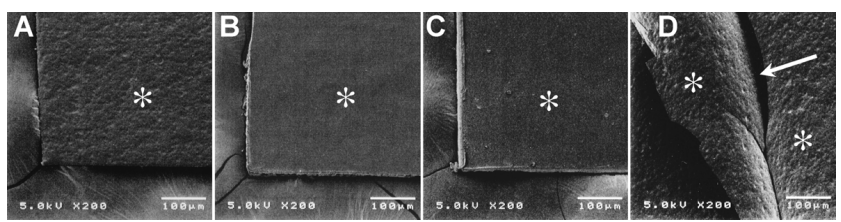

Fig. 5. SEM Photographs of SC Film Containing GL (F-GL 0.25\%, A), ST (F-ST $0.25 \%$, B), PG (F-PG $0.25 \%$, C), and Not Containing Any Plasticizer (F-NC, D) at 200-Fold Magnification

Scale bar shows $100 \mu \mathrm{m}$. * Surface of film. Arrowhead: crack in film. sis showed that the secondary structure of SC in dried SC film, and in solution comprised of SC alone, is largely random coil (IR cm $\left.{ }^{-1}: 1641\right){ }^{23,27)}$ Moistened SC film and gel were reported to exhibit $\beta$-sheet structure (IR $\mathrm{cm}^{-1}$ : $1618) .^{23,27)}$ In addition, the tensibility of moistened SC film was improved compared with dried film. ${ }^{22}$ In the present ATR-FT-IR analysis of SC films, the peak around $1618 \mathrm{~cm}^{-1}$ became higher and sharper if GL, ST or PG were added (Fig. $6)$. These results suggest that the secondary structure of SC in films containing GL, ST or PG was changed to $\beta$-sheet structure, as with moistened SC film and gel.

Figure 7 shows the elongation and the tensile strength of

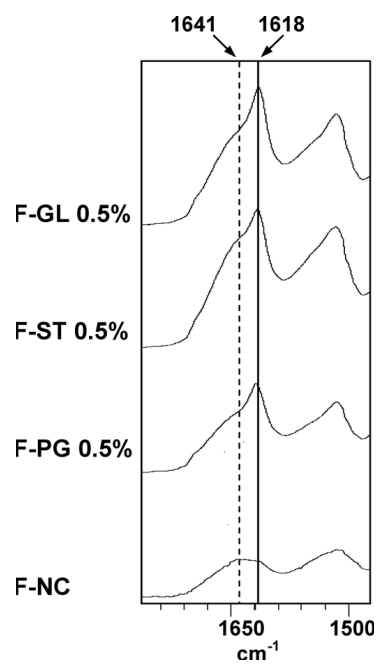

Fig. 6. ATR-FT-IR Spectra of SC Film Containing GL (F-GL 0.5\%), ST (F-ST 0.5\%), PG (F-PG 0.5\%), and Not Containing Plasticizer (F-NC)

The solid line shows IR $\mathrm{cm}^{-1}$ : around 1618 . The broken line shows IR $\mathrm{cm}^{-1}$ : around 1641.
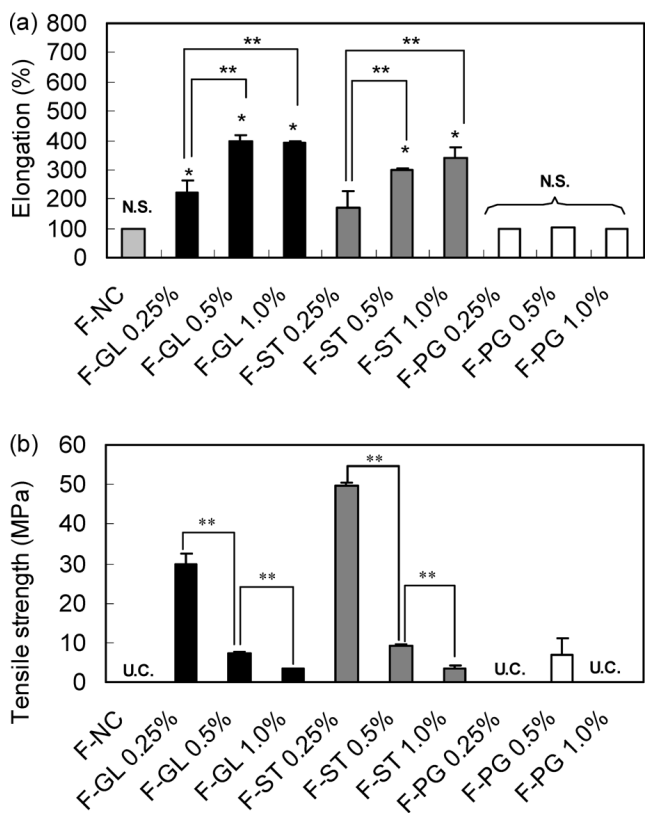

Fig. 7. Elongation (a) and Tensile Strength (b) of Dried SC Films Containing Various Concentrations of GL (F-GL $0.25 \%$, F-GL $0.5 \%$ and F-GL $1.0 \%$ ), ST (F-ST $0.25 \%$, F-ST $0.5 \%$ and F-ST $1.0 \%$ ), or PG (F-PG $0.25 \%$, F-PG $0.5 \%$ and F-PG $1.0 \%$ ), and Not Containing Plasticizer (F-NC)

N.S., not stretched; U.C., unable to carry out. Each bar represents the mean \pm S.D. $(n=3)$. Statistical comparison was made by two-sided $t$-test $(a=0.05) . * p<0.05$ : significant difference versus $\mathrm{F}-\mathrm{NC}$, $* * p<0.05$. 


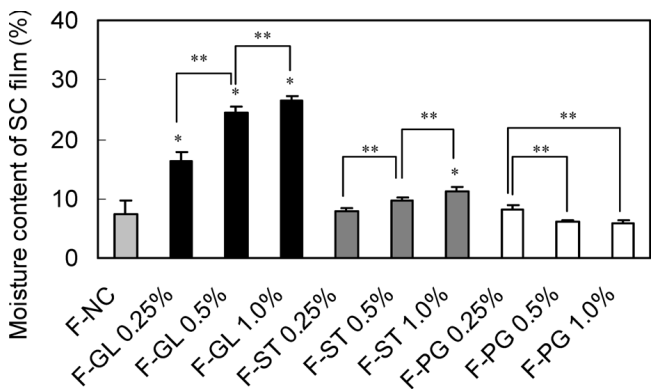

Fig. 8. Moisture Content of Dried SC Films Containing Various Concentrations of GL (F-GL $0.25 \%$, F-GL $0.5 \%$ and F-GL $1.0 \%$ ), ST (F-ST $0.25 \%$, F-ST $0.5 \%$ and F-ST $1.0 \%$ ), or PG (F-PG $0.25 \%$, F-PG $0.5 \%$ and F-PG $1.0 \%$ ), and Not Containing Any Plasticizer (F-NC)

Statistical comparison was made by two-sided $t$-test $(a=0.05) . * p<0.05$ : significant difference versus $\mathrm{F}-\mathrm{NC}$, ** $p<0.05$.

dried SC films containing GL, ST or PG. The elongation of dried SC films tended to increase up to $170-400 \%$ with increasing GL and ST content. These results suggest that the allowable elongation of SC film containing GL or ST can be up to $400 \%$ (Fig. 7a). On the other hand, the tensile strength decreased with increasing GL and ST content (Fig. 7b). Both the film-forming characteristics and tensibility of SC films were improved by the addition of GL or ST. Even $0.25 \%$ GL or ST in SC films improved the film-forming properties and tensibility sufficiently to make the films practical for use. In contrast, SC films containing PG tore easily and were difficult to stretch (Fig. 7a), even though the film-forming properties were improved (Fig. 5C). The SC films containing PG (F-PG $0.25 \%$ and F-PG $1.0 \%$ ) or not containing plasticizer (F-NC) could not be tested due to the weakness of the films.

The moisture content of dried SC films containing GL, ST or PG was measured (Fig. 8). The addition of GL or ST enhanced moisture retention by SC film. In contrast, the addition of PG tended to decrease the moisture content of SC film. The moisture content was found to correlate with the elongation of SC film. Thus, to obtain a practical film with good film-forming characteristics and tensibility, the SC should be in $\beta$-sheet conformation and the film should have sufficient moisture content; this can be achieved by adding $0.25-0.5 \%$ GL or ST.

The elongation properties of SC film moistened with PBS tended to decrease at the highest content of each plasticizer studied, although there was no significant difference in elongation at any concentration of GL between 0.25 and $0.5 \%$ ST, or between 0.25 and $0.5 \%$ PG (Fig. 9a). The tensile strength of these films tended to decrease with increasing content of plasticizer, although there was no significant difference between 0.25 and $0.5 \%$ ST (Fig. 9b). These results suggest that the plasticizers dissolved from the moistened SC film in PBS and caused the film to swell, resulting in low mechanical strength. Thus, adding a lower concentration of GL or ST $(0.25 \%)$ is more useful for preparing both moistened and dry tensible film for practical use in both the moistened and dried condition.

Release Profiles of Model Drugs from SC Gel, Sponge and Film To evaluate the release properties, SC gel, sponge and film were prepared in cylindrical glass vessels (Table 3). The release rates of the three model drugs (FD4, FD70 and FA) from SC preparations in PBS (pH 7.4) were
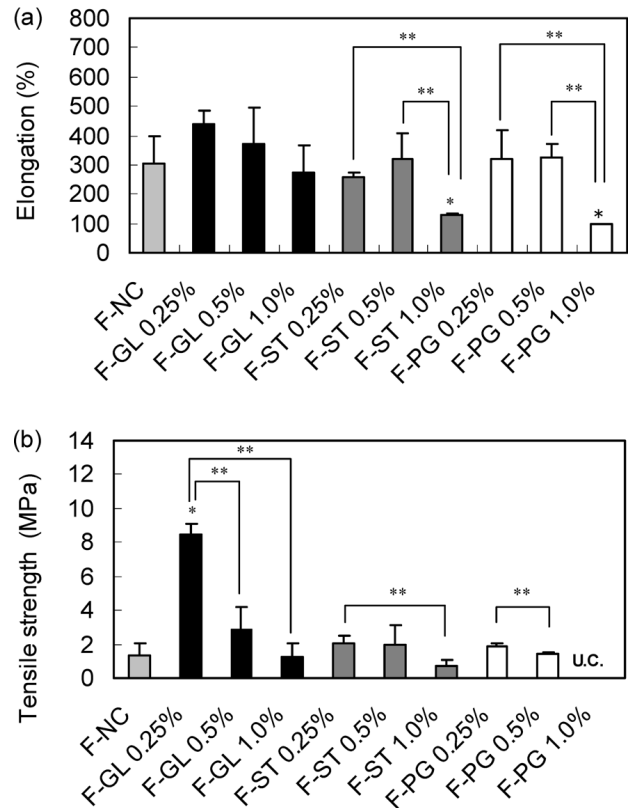

Fig. 9. Elongation (a) and Tensile Strength (b) of Moistened SC Films Containing Various Concentrations of GL (F-GL $0.25 \%$, F-GL $0.5 \%$ and FGL $1.0 \%$ ), ST (F-ST $0.25 \%$, F-ST $0.5 \%$ and F-ST $1.0 \%$ ), or PG (F-PG $0.25 \%$, F-PG $0.5 \%$ and F-PG $1.0 \%$ ), and Not Containing Any Plasticizer (F$\mathrm{NC)}$

SC films were moistened with PBS (pH 7.4). U.C.: unable to carry out. Each bar represents the mean \pm S.D. $(n=3)$. Statistical comparison was made by two-sided $t$-test $(a=0.05) . * p<0.05$ : significant difference versus $\mathrm{F}-\mathrm{NC}, * * p<0.05$.

measured. The original shapes of the SC preparations were maintained in the early stages of the dissolution test, then gradually disintegrated or eroded during further incubation in the dissolution medium. In the model structure of hydrated $\mathrm{SC}$ generated from ${ }^{13} \mathrm{C}$ solid state NMR data, Ser and Thr linkages are involved in hydrophobic $\beta$-sheet and random coil structure formation in the hydrated state, and the Asnrich sequences form a hydrophilic region. ${ }^{27)}$ In the degradation or erosion of SC preparations, the hydrophilic regions containing Asp residues in the sequence could be the cause of the easy hydrolytic degradation. ${ }^{24}$

Figure 10 shows the release profiles of FD4, FD70 and FA from each SC preparation. The release rate of the model drugs tended to be FA $<$ FD70 $<$ FD4. Large molecular weight FD70 $(70 \mathrm{kDa})$ was released more slowly than small molecular weight FD4 $(4 \mathrm{kDa})$ from SC film. FA (66 kDa) was released the slowest, for a period of 1 week or more from each preparation (Figs. 10a-c), even though the molecular weights of FA and FD70 are almost the same. Kang and Song reported that, in their study of the release of drugs from chitosan, negatively charged FA was easily bound to amino groups in chitosan, allowing the sustained release of FA from a chitosan hydrogel. ${ }^{28)}$ The isoelectric point (pI) of FA is 4.7-4.9, so FA is negatively charged in PBS ( $\mathrm{pH}$ 7.4). On the other hand, FITC-dextran is an uncharged compound. Amino acid composition analysis of SC by the PTC method showed that SC contains 18 different amino acids, of which about $70 \%$ are polar amino acids. Of these $70 \%$, about $40 \%$ are neutral amino acids like Ser and Thr, about $20 \%$ are acidic amino acids like Asp/Asn and Glu/Gln, and about $10 \%$ are basic amino acids like Lys, Arg and His. ${ }^{2,16)}$ Two-dimensional electrophoresis indicates that the $\mathrm{pI}$ of SC in solu- 

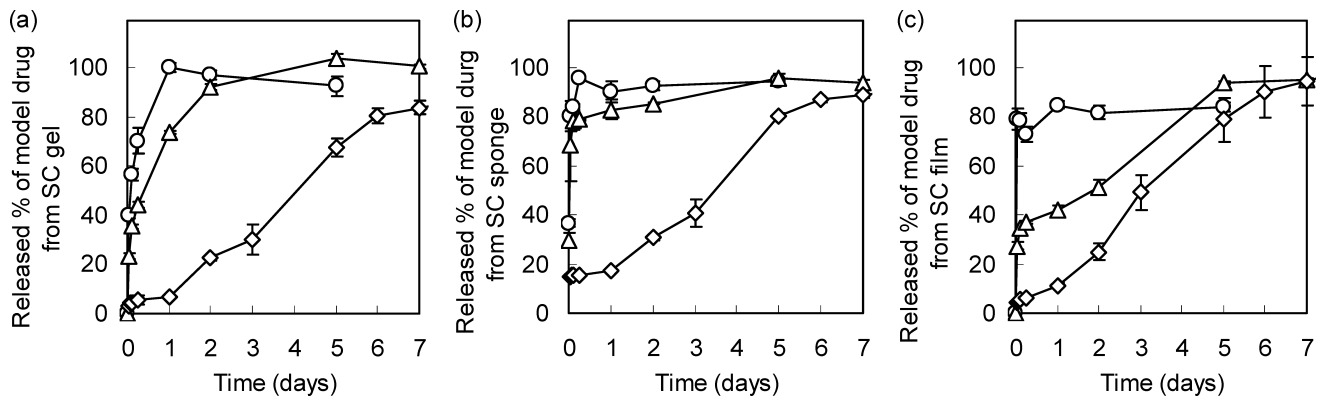

Fig. 10. Release Profiles of FD4 $(\bigcirc)$, FD70 $(\triangle)$ and FA $(\diamond)$ from SC Gel (a), Sponge (b), or Film (c) in PBS (pH 7.4$)$ at $37^{\circ} \mathrm{C}$ Each bar represents the mean \pm S.D. $(n=3)$.
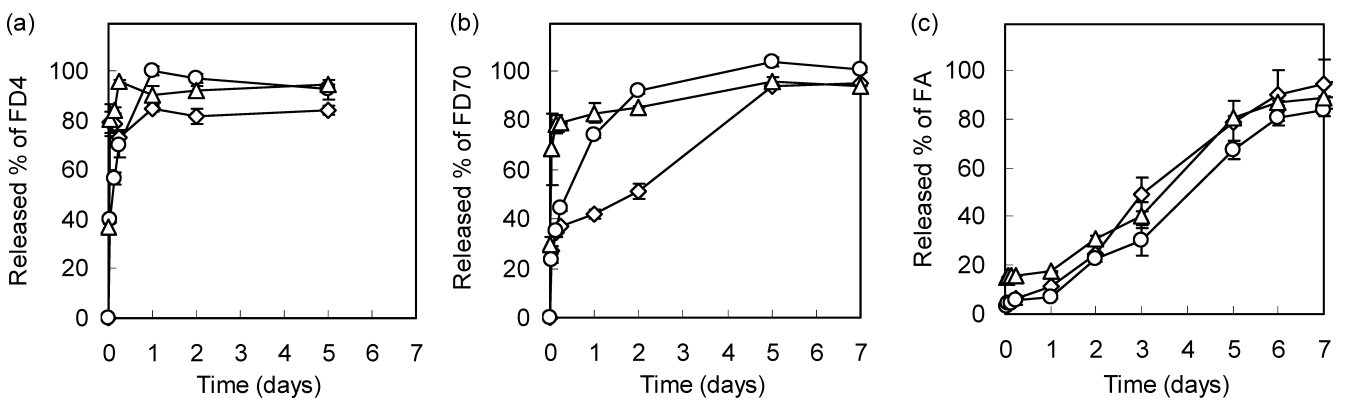

Fig. 11. Release Profiles of FD4 (a), FD70 (b) and FA (c) from SC gel (O), sponge ( $\triangle$ ), or film $(\diamond)$ in $\mathrm{PBS}(\mathrm{pH} 7.4)$ at $37^{\circ} \mathrm{C}$

Each bar represents the mean \pm S.D. $(n=3)$.

tion is between 5 and $6{ }^{29)}$ suggesting that SC is also negatively charged at $\mathrm{pH}$ 7.4. However, the secondary structure of SC changes from hydrophilic random coil conformation in solution to hydrophobic $\beta$-sheet structure in gel or moistened film. ${ }^{23)}$ Structural analysis of the Ser1 gene coded major SC proteins (S1 proteins) indicated that S1 proteins consist of the repetitive subunits of 8 peptides. The large peptides (peptides 6 and 8 ) are rich in Ser (40\%) and form hydrophobic $\beta$ sheet structures through hydrogen bonds between the $\mathrm{OH}$ groups of the Ser and Thr side chains and the $\mathrm{C}=\mathrm{O}$ groups of the backbone of adjacent strands. ${ }^{6,27)}$ On the other hand, $\mathrm{SC}$ has a hydrophilic region (peptide 3 ) containing charged residues $(\mathrm{Glu}+\mathrm{Asp}=13 \%$, and Lys $+\mathrm{Arg}=15 \%)$ between the hydrophobic sequence and the $\beta$-sheet. $\left.{ }^{6}\right)$ In this study, because SC was in the solid state as a gel, sponge and film in PBS ( $\mathrm{pH} 7.4$ ), the SC preparations were expected to form $\beta$ sheet structures and to contain charged residues in the hydrophilic region, analogous to peptide 3 . We therefore believe that negatively charged FA interacts ionically with positively charged residues like Lys (pI: 9.75) and Arg (pI: 10.76) in SC preparations in PBS ( $\mathrm{pH} 7.4$ ), thereby sustaining longer release of FA from SC preparations compared to uncharged FD4 and FD70.

Figure 11 shows the effect of the SC form (gel, sponge or film) on the release of each model drug. FD4 (a) was released rapidly from each SC preparation. The release rate of FD70 (b) was film $<$ gel $<$ sponge. There was no significant difference in the release rate of FA (c) from the three SC preparations.

Of the three SC preparations, the sponge form disintegrated most easily during the dissolution test. SEM observation of SC film showed that the surfaces of SC films were smooth, and no pores were evident in film cross-sections

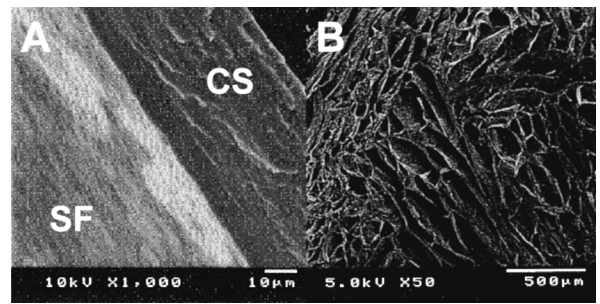

Fig. 12. SEM Photographs of SC Film (A) and Freeze-Dried Sponge (B) Made from SCS-1, at 1000-Fold or 50-Fold Magnification

Scale bar shows $10 \mu \mathrm{m}$ at 1000 -fold magnification in A and $500 \mu \mathrm{m}$ at 50 -fold magnification in B. SF, surface of film; CS, cross-section of film.

(Fig. 12A). On the other hand, many pores were observed in SC sponge (Fig. 12B). These observations suggest that drugs might easily diffuse from the pores in SC sponge into the dissolution medium. In SC gel, SC adopts a $\beta$-sheet structure that constructs the network structure of the hydrogel. ${ }^{6,23)}$ This allows the gel to retain a large amount of water, possibly allowing larger molecular weight drugs like FD70 to be retained in the gel network structure. In the case of drugs that interact ionically with the carrier, the diffusion of the drug may be inhibited, judging from our observation that the initial release rate of FA from SC preparations was markedly slower compared to that of FD70 or FD4.

The present results suggest that SC gel, sponge and film can be used as a sustained-release material for drugs with high molecular weight, and if these drugs carry a charge, their release rate is slower and more prolonged.

\section{Conclusion}

The molecular weight of SC tended to decrease with increasing temperature and heating time used to dissolve the 
SC. The gel and sponge formed from high molecular weight SC $(250 \mathrm{kDa}$ and about $400 \mathrm{kDa})$ were moldable. The film made of SC alone was easily broken and exhibited elastic distortion. By adding a moisture-retaining plasticizer (GL and ST), the film-forming characteristics of SC were improved, the secondary structure of the protein changed, and the tensibility of the film was also improved when the moisture content of the film was increased. The results suggest that $\mathrm{SC}$ is usable and practical as a moldable gel, sponge and tensible film.

In each preparation, the release rate of the model drugs tended to be FA $<$ FD70 $<$ FD4. FA, which has a large molecular weight and is negatively charged, was released for the longest period of time ( $\geq 1$ week) from each preparation. The results indicate that $\mathrm{SC}$ is useful as an aqueous sustained-release material for large molecular weight drugs, and if drugs are charged, their release can likely be sustained for a still longer period of time.

Acknowledgments The authors thank Mr. Kazuma Hirata and Mr Kazumasa Yokoyama for valuable advice on the experimental, and Mr. Minoru Terashima for helpful advice on the manuscript.

\section{References}

1) Zhang Y.-Q., Biotechnol. Adv., 20, 91-100 (2002).

2) Takasu Y., Yamada H., Tsubouchi K., Biosci. Biotechnol. Biochem., 66, 2715-2718 (2002)

3) Okamoto H., Ishikawa E., Suzuki Y., J. Biol. Chem., 257, 15192 15199 (1982)

4) Gamo T., Biochem. Gene., 20, 165-177 (1982).

5) Michaille J.-J., Garel A., Prudhomme J.-C., Gene, 86, 177-184 (1990).

6) Garel A., Deleage G., Prudhomme J.-C., Insect Biochem. Molec. Biol., 27, 469-477 (1997).

7) Ogawa S., Tomita M., Shimizu K., Yoshizato K., J. Biotechnol., 128, $531-544$ (2007)
8) Tomita M., Hino R., Ogawa S., Iizuka M., Adachi T., Shimizu K., Sotoshiro H., Yoshizato K., Transgenic Res., 16, 449- 465 (2007).

9) Based on a Lecture Presented at the 2009 Silk Summer Seminar at the National Institute of Agrobiological Sciences, Okaya.

10) Takabayashi C., Takezawa T., Japanese Patent, No. 3840541.

11) Tsubouchi K., Igarashi Y., Takasu Y., Yamada, H., Biosci. Biotechnol. Biochem., 69, 403-405 (2005).

12) Teramoto H., Kameda T., Tamada Y., Biosci. Biotechnol. Biochem., 72 $3189-3196$ (2008)

13) Tsubouchi K., Japanese Patent No. 11-070160A (1999).

14) Dash R., Acharya C., Bindu P., Kundu S. C., BMB Reports, 41, 236 241 (2008)

15) Kato N., Sato S., Yamanaka A., Yamada H., Fuwa N., Nomura M. Biosci. Biotechnol. Biochem., 62, 145-147 (1998).

16) Dash R., Mandal M., Ghosh S. K., Kundu S. C., Mol. Cell Biochem., 311, 111-119 (2008).

17) Okada H., Doken Y., Ogawa Y., Toguchi H., Pharm. Res., 11, 11431147 (1994).

18) Kanazawa T., Takashima Y., Murakoshi M., Nakai Y., Okada H., Int. J. Pharm., 379, 187-195 (2009).

19) Kushibiki T., Tomoshige R., Iwanaga K., Kakemi M., Tabata Y., J. Controlled Release, 112, 249-256 (2006)

20) Bhattarai N., Gunn J., Zhang M., Adv. Drug Delv. Rev., 62, 83-99 (2010).

21) Tao W., Li M., Xie R., Macromol. Mater. Eng., 290, 199-194 (2005).

22) Teramoto H., Miyazawa M., Biomacromolecules, 6, 2049-2057 (2005).

23) Teramoto H., Nakajima K., Takabayashi C., Biosci. Biotechnol. Biochem., 69, 845-847 (2005).

24) Teramoto H., Kakazu A., Asakura T., Macromolecules, 39, 6-8 (2006).

25) Mase K., Iizuka T., Okada E., Miyajima T., Yamamoto T., J. Insect Biotechnol. Sericol., 75, 85-88 (2006).

26) Jackson M., Mantsch, H. H., Crit. Rev. Biochem. Molec. Biol., 30 , 95-120 (1995).

27) Teramoto H., Kakazu A., Yamauchi K., Asakura T., Macromolecules, 40, 1562-1569 (2007)

28) Kang G. D., Song S.-C., Int. J. Pharm., 349, 188-195 (2008).

29) Capar G., Aygun S.-S., Gecit M.-R., J. Membrane Sci., 325, 920-931 (2008). 